\title{
Characterization of colistin-resistant $A$. baumannii isolated in Intensive Care Unit of an Italian Hospital
}

\author{
Erika Coppo', Pier Andrea Dusi², Monica Dotta ${ }^{2}$, Eugenio A. Debbia ${ }^{3}$, Anna Marchese ${ }^{3}$ \\ I Sezione di Microbiologia DISC, Università di Genova, \\ 2 ASL I Imperiese, Ospedale di Sanremo \\ 3 IRCCS-san Martino IST Genova, Sezione di Microbiologia DISC, Università di Genova
}

Key words: Acinetobacter, Colistin, PmrB

\section{SUMMARY}

We report the characterization of an Acinetobacter baumannii resistant to colistin isolated from a patient treated with colistin for 22 days.

The identification and initial susceptibility testing of the strain was performed at ASL3 Imperiese with the Vitek-2 automated system and than the strain was re-identified at the Sezione di Microbiologia with APINE. In vitro activity of antimicrobial agents was determined by the microdiluition methods. The detection of the beta-lactamase gene was performed by PCR and for the analysis of LPS were sequenced the genes of the PmrABC system.

We compared the PFGE profile of the colistin-resistant A. baumannii with 5 A. baumannii colistin-susceptible strains isolated in the same Hospital during 2010.

The strain was resistant to all antimicrobial agents tested, except to tygecyclin. The PFGE shown that the A. baumannii colistin-resistant was closery related to the colistin-susceptible strains.

The sequencing of the PmrABC system showed that the strain had a single mutation in the PmrB component. Colistin-resistant strain have recently been found in several Gram-negative bacteria such A. baumannii, $K$. pneumoniae and P. aeruginosa.

This clinical case confirms that resistance to colistin in A. baumannii can be selected in vivo following the use of colistin in therapy.

\section{INTRODUCTION}

Acinetobacter baumannii is an important pathogen in Intensive Care Unit (ICU) patients. In nosocomial $A$. baumannii isolates resistance to extended-spectrum beta-lactams, carbapenems, aminoglycosides and fluoroquinolones are widespread. Colistin is the last resort for treatment of MDR A. baumannii, unfortunally, however, resistance to colistin has been reported all over the word. The mechanism of resistance is not yet clear, but some study confirm that it might be related to a loss of LPS $(12,13,7)$ or/and the PmrAB two component system $(1,14)$.

We report the characterization of a colistin-resistant $A$. baumannii isolated from a 85 years old man after colistin therapy.

We compared the PFGE profile of the colistinresistant $A$. baumannii with those of other $A$. baumannii strains isolated in this geographic area.

\section{Bacterial strains and patients}

The patient was transferred on $08 / 31 / 11$ to ASL3 Imperiese Hospital from the ICU of Santa Corona Hospital where he was hospitalized following chest trauma with multiple injuries. At the admission to ASL3 the patient was already tracheostomized.

On day 1 , a colistin-susceptible $A$. baumannii isolate (310811) was recovered from broncho-lavage, the patient was treated with voriconazole and piperacillin/tazobactam. On day 13 a throat swab was positive to the colistin-susceptible $A$. baumannii, while broncho-lavage was negative. The treatment was switched to meropenem, voriconazole and colistin. On day 20, a throat swab was positive to the colistin-susceptible $A$. baumannii, while broncho-lavage was negative. The treatment was switched to meropenem, ciprofloxacin and colistin. On day 35 a colistin-resistant $A$. baumannii isolate (051011) was recovered from broncho-lavage. A colistin resistant $A$. baumannii was also present in broncho-lavage by day 43 and in throat swobs and in broncho-lavage by day 50 . On day 53 the patient died.

A. baumannii (310811), A. baumannii (051011) and 10 colistin-susceptible $A$. baumannii isolated at ASL3 Imperiese Hospital in 2010 from different clinical sample (blood, broncho-lavage, bron-

\section{Corresponding author: Erika Coppo}

Sezione di Microbiologia DISC, Università degli Studi di Genova - Largo R.Benzi 10, 16132 Genova

Tel: +3901035338136 - Fax:+39010353765I;

E-mail: erika.coppo@unige.it 
cho-aspirate, catheter tip and oral swab) were characterized by pulsed-field gel electroforesis.

\section{METHODS}

The identification and initial susceptibility testing of $A$. baumannii isolate was performed with the Vitek-2 automated system (bioMérieux, France). The strain was sent to Sezione di Microbiologia, University of Genoa for further investigation.

The strain was re-identified with API NE (bioMérieux, France). In vitro activity of ampicillin, amoxicillin/clavulanate, piperacillin/tazobactam, ceftazidime, imipenem, gentamicin, ciprofloxacin, trimethoprim-sulfamethoxazole and tigeciclyne was determined by the broth microdiluition methods and interpreted according to the Clinical Laboratory Standard Institute (CLSI) (5). In addition, MIC of colistin was determined by the agar diluition (11) and interpreted using the European Committee on Antimicrobial Susceptibility Testing (EUCAST) criteria (6).

The detection of beta-lactamase gene was performed by PCR, using previously condition and primers for blaOXA51, blaOXA23, blaOXA24 and blaOXA58 (18).

The genetic relatedness of $A$. baumannii colistinresistant (051011), susceptible isolated from BAL (310811) and 5 A. baumannii isolates in 2010, was determined by molecular typing using pulsed-field gel electrophoresis (PFGE) as follows: genomic DNA, extracted and digested with ApaI (New England B Biolabs Inc., MA, USA) as previously described (16), was separated by PFGE using CHEF DRII device (Bio-rad, Hercules, CA, USA). Running conditions were as follows: $27 \mathrm{~h}$ at $11.3^{\circ} \mathrm{C}$ at voltage of $5.4 \mathrm{~V}$, ramped with an initial forward time of $5 \mathrm{~s}$ and final forward time of 40s. After electrophoresis, gel was stained with ethidium bromide $(1 \mu \mathrm{g} / \mathrm{ml})$ and visualized on an ultraviolet transilluminator.

The banding patterns were interpreted visually following published guidelines (17).

Primer and conditions previously described (3) were used for sequencing the PmrAB two components system. The amplicons were purified using High Pure PCR Cleanup MicroKit (Roche) and sequenced as previously described (4).

\section{RESULTS}

The isolate showed a multidrug-resistant phenotype, being susceptible only to tigecycline. MIC to colistin was $16 \mu \mathrm{g} / \mathrm{ml}$.

The strain was positive for blaOXA51 and bla OXA23 by PCR.

The PFGE of ApaI digested genomic DNA of $A$. baumannii colistin-resistant and $A$. baumannii colistin-susceptible and of $5 \mathrm{~A}$. baumannii colis- tine susceptible isolate from the same ward in 2010 revelated that $A$. baumannii colistin-resistant and colistin-susceptible were closely related, this clone has been found circulating in this hospital since 2010 .

The colistin-resistant isolate (051011) had a single aminoacid change in PmrB (Pro233Ser). No differences were found in the $p m r A$ or $p m r C$ gene.

\section{DISCUSSION}

Colistin-resistant isolates have recently been identified in several Gram-negative bacterial species, such $A$. baumannii, $K$. pneumoniae and $P$. aeruginosa $(2,8$, 9). In 2011 Kempf-Rolain (15) and López-Rojas (10) reported in France and in Spain, respectively, two cases of patients with $\mathrm{A}$. baumannii becoming resistant to colistin after colistin therapy. Our case report confirms that colistinresistant A. baumannii strain may be selected in vivo by the use of colistin.

The results of PFGE indicated a unique epidemic clone circulating in ASL3 Imperiese Hospital, where all strains, including the A. baumannii colistin-resistant were closely related. The presence of a unique clone persistently occurring in an ICU is also connected to the capability of the microrganism to survive for long periods in the hospital environment, even in dry conditions.

\section{REFERENCES}

1. Adams MD, Nickel GC, Bajaksouzian S, et al. Resistance to colistin in Acinetobacter baumannii associated with mutations in the PmrAB two-component system. Antimicrob Agents Chemother 2009; 53: 3628-34.

2. Antoniadou A, Kontopidou F, Poulakou G, et al. Colistinresistant isolates of Klebsiella pneumoniae emerging in intensive care unit patients: first report of multiclonal cluster. J Antimicr Chemother 2007; 60: 1163-7.

3. Beceiro A, Llobet E, Aranda J, et al. Phosphoethanolamine modification of Lipid A in colistin-resistant variants of Acinetobacter baumannii mediated by the pmrAB two-component regulatory system. AAC, 2011; 3370-9.

4. Cagnacci S, Gualco L, Roveta S, et al. Bloodstream infections caused by multidrug resistant Klebsiella pneumoniae producing the carbapenem-hydrolysing VIM-1 metallo-beta-lactamase: firs Italian outbrake. $J$ Antimicrob Chemother 2008; 61 (2): 296-300.

5. Clinical and laboratory Standards Institute. Performance standards for antimicrobial susceptibility testing:Twenty-Second Informational Supplement. DocumentM100-S22. Wayne, PA: CLSI; 2012.

6. European Committee on Antimicrobial Susceptibility Testing. Break-point tables for interpretation of MICs and zone diameters, version 2.0. Basel: EUCAST, January 2012.

7. Henry R, Vithanage N, Harrison P, et al. Colistinresistant, lipopolysaccharide-deficient Acinetobacter baumannii responds to lipopolysaccharide loss through increased expression of genes involved in the synthesis and transport of lipoproteins, phospholipids, 
and poly-b-1,6-N-acetylglucosamine. Antimicrob Agents Chemother 2012; 56: 59-69.

8. Johansen HK, Moskowitz SM, Ciofu O, Pressel T, Høiby N. Spread of colistin resistant non mucoid Pseudomonas aeruginosa among clinically infected danish cystic fibrosis patients. $J$ Cyst Fibros 2008; 7 : 391-7.

9. Ko KS, Suh JY, Kwon KT, et al. Hight rates of resistance to colistin and polimixin B in subgroups of Acinetobacter baumanii isolates from Korea. $J$ Antimicrob Chemother 2007; 60: 1163-7.

10. López-Rojas R, Jimenéz-Mejías ME, Lepe JA, Pachón J. Acinetobacter baumannii resistant to colistin alters its antibiotics resistance profile: a case report from spain. $J$ Infect Dis 2011; 204: 1147-8.

11. Lo-Ten-Foe JR, De Smet AM, Diederen BM, Kluytmans JA, van Keulen PH. Comparative evaluation of the Vitek2, disk diffusion, Etest, broth microdiluition, and agar diluition susceptibility testing methods for colistin in clinical isolates, including heteroresistant Enterobacter cloacae and Acinetobacter baumannii strains. Antimicrob. Agents Chemother. 2007; 51: 3726-30.

12. Moffatt JH, Harper M, Harrison P, et al. Colistin resistance in Acinetobacter baumannii is mediated by complete loss of lipopolysaccharide production. Antimicrob Agents Chemother 2010; 54: 4971-7.
13. Moffatt JH, Harper M, Adler B, et al. Insertion sequence ISAba11 is involved in colistin resistance and loss of lipopolysaccharide in Acinetobacter baumannii. Antimicrob Agents Chemother 2011; 55: 3022-4.

14. Park YK, Choi JY, Shin D, et al. Correlation between overexpression and amino acid substitution of the PmrAB locus and colistin resistance in Acinetobacter baumannii. Int J Antimicrob Agents 2011; 37: 525-30.

15. Rolain JM, Roch A, Castainer M, Papazian L, Raoult D. Acinetobacter baumanii resistant to colistin with impaired virulance: a case report from France. $J$ Infect Dis 2011; 204: 1146-7.

16. Seifert H, Dolzani L, Bressan R, et al. Standarization and interlaboratory reproducibility assestement of Pulsed-Field gel electroforesis- generated fingerprints of Acinetobacter baumannii. JCM sept 2005; 432835 .

17. Tenover FC, Arbeit RD, Goering RV, et al. Interpreting chromosomal DNA restriction patterns produced by pulsed-field gel electrophoresis: criteria for bacteril strain typing. J Clin Microbiol 1995; 33: 2233-9.

18. Woodford N, Ellingtona MJ, Coelhoa JM, et al. Multiplex PCR for genes enconding prevalent OXA carbapenemase in Acinetobacter spp. Int J Antimic Ag. April 2006; 27 (4): 351-3. 